\title{
Mental Health Services in South Africa: Taking stock
}

\author{
C Lund', I Petersen², S Kleintjes', A Bhana ${ }^{3}$ \\ ${ }^{1}$ Alan J Flisher Centre for Public Mental Health, Department of Psychiatry and Mental Health, University of Cape Town, South Africa \\ 2School of Psychology, University of KwaZulu-Natal, South Africa \\ 3Human Sciences Research Council of South Africa, South Africa
}

\begin{abstract}
There is new policy commitment to mental health in South Africa, demonstrated in the national mental health summit of April 2012 . This provides an opportunity to take stock of our mental health services. At primary care level key challenges include- training and supervision of staff in the detection and management of common mental disorders, and the development of community-based psychosocial rehabilitation programmes for people with severe mental illness (in collaboration with existing non-governmental organizations). At secondary level, resources need to be invested in 72-hour observation facilities at designated district and regional hospitals, in keeping with the Mental Health Care Act. At tertiary level, greater continuity of care with primary and secondary levels is required to prevent "revolving door" patterns of care. There are major challenges and also opportunities related to the high level of comorbidity between mental illness and a range of other public health priorities, notably HIV/AIDS, cardiovascular disease and diabetes. The agenda for mental health services research needs to shift to a focus on evaluating interventions. With current policy commitment, the time to act and invest in evidence-based mental health services is now.
\end{abstract}

Keywords: Mental health services; Public health; Mental health policy; South Africa

Received: 03/07/2012

Accepted: 17/07/2012

doi: http://dx.doi.org/10.4314/ajpsy.v15i6.48

This article is based on a paper presented at the National Mental Health Summit convened by the Department of Health, Republic of South Africa from 12-13 April 2012.

\section{Introduction \\ On the historic occasion of the first national mental health summit in South Africa, it is important to take stock of our mental health services. This is necessary for four reasons. First, the burden of mental illness in South Africa is substantial, and is likely to increase with the epidemiological transition to chronic and non- communicable diseases - a pattern common to many low and middle-income countries. ${ }^{1}$ Mental disorders rank third in their contribution to the burden of disease in this country ${ }^{2}$, and approximately $l$ in 6 South Africans are likely to experience a common mental disorder (depression, anxiety or substance use disorder) during the current year. ${ }^{3}$ \\ Second, the relationship between physical and mental health is well established, particularly with respect to HIV/AIDS,}

Correspondence
Prof C Lund
Alan J Flisher Centre for Public Mental Health, Department of Psychiatry
and Mental Health, University of Cape Town, 46 Sawkins Road,
Rondebosch, 7700, South Africa.
email: crick.lund@uct.ac.za

cardiovascular disease and diabetes. ${ }^{4}$ With the transition of HIV/AIDS to a chronic illness, common mental disorders (CMDs) in people living with HIV/AIDS (PLWHAs) are of particular concern in South Africa. Depression and alcohol misuse, which are more prevalent in PLWHAs, compromise adherence to antiretroviral therapy (ART) and virological suppression. . $^{5-8}$

Third, a staggering $75 \%$ of people who live with mental disorders in South Africa do not receive the care that they need. ${ }^{3}$ To address this growing burden and narrow the treatment gap, we need to develop evidence-based and culturally appropriate mental health services that can feasibly be delivered within available resource constraints and integrated with other health services, particularly for HIV.

Fourth, there appears to be a growing policy impetus to improve mental health services in South Africa, since the demise of apartheid. In 1997, mental health was included in the White paper for the transformation of the health system in South Africa, which stated: "a comprehensive and community-based mental health service should be planned and coordinated at the national, provincial, district and community levels, and integrated with other health services" p136. ${ }^{9}$ Subsequently the Mental Health Care Act no 17 of 2002 was promulgated, which enshrined the human rights of people living with mental disorders, and set up mechanisms such as Mental Health Review Boards, to protect and uphold those rights. Most recently, through the convening of a national mental health summit on 12-13 April 2012, the national 
Minister of Health, Dr Aaron Motsoaledi has committed himself and the Department of Health to redressing the past neglect of mental health care. This is encouraging for the many people in South Africa who suffer from mental disorders, and the service providers who work in the health and mental health sector.

This impetus must be supported by an accurate assessment of the current status of South Africa's mental health services. The purpose of this article is to review current mental health service provision in South Africa, in order to make recommendations for service development. The review draws on two principle sources: the first is a systematic review of mental health services research in South Africa, conducted between 2000 and 2010 10; and the second is a national survey of mental health services. ${ }^{11}$ The methodology and analysis used in these studies are set out in the respective articles. Both of these studies were conducted as part of the Mental Health and Poverty Project, a collaboration between academic researchers and the Department of Health during 2005-2010.12

\section{Levels of care}

Mental health services are delivered broadly at primary, secondary and tertiary levels in South Africa. We will therefore structure this review according to these levels, and then examine some cross cutting themes that pertain to all three levels.

\section{- Primary care}

At this level, management for people with severe mental disorders such as schizophrenia and bipolar mood disorder consists mainly of symptom management through provision of follow-up medication. A typical scenario for many South Africans living with these disorders is that they experience a first episode of illness, frequently in early adulthood, and are admitted to either a secondary or tertiary facility for acute management of their condition. Once stabilized they are discharged to the community, and referred to primary care clinics, which they are expected to attend to receive medication and monitoring of their mental health status on ongoing basis. In most instances, primary care facilities and staff are not well equipped to address these needs. Although primary care nurses are reported by some studies to be comfortable with prescription maintenance of chronic severe mental disorders, psychotropic medication is not universally available at primary care clinics. ${ }^{11}$

Beyond medication monitoring, there is little communitybased psychosocial rehabilitation (PSR) at this level in South Africa, and several studies have highlighted shortcomings in this regard. ${ }^{10}$ PSR is largely offered by non-governmental organizations (NGOs) affiliated to the South African Federation for Mental Health, which face ongoing challenges due to inadequate funding from government. ${ }^{13}$ Research has shown that the intensity, specificity and length of community-based PSR services were related to superior functional outcomes for individuals diagnosed with schizophrenia. ${ }^{14}$ Without community-based rehabilitation, mental health care users (MHCUs) are likely to find it difficult to reintegrate into their communities, and have little support for their recovery. They are therefore at high risk for defaulting on clinic appointments and medication, and are more likely to require readmission. There is potential for MHCUs to be more actively involved in peer support and advocacy, with the appropriate level of support. ${ }^{15}$ Assertive community-based treatment (ACT) has been found to be effective for reducing readmissions and improving social and occupational functioning in high frequency service users in the Western Cape. ${ }^{16}$ MHCUs in some studies reported a lack of continuity of care and would prefer a dedicated psychiatric service over an integrated service at primary care clinic level. ${ }^{17,18}$

In relation to the detection and management of common mental disorders (CMD) at primary care level, there is a large treatment gap, and identification and treatment is irregular and inconsistent. 11,19-21 This is reported to be a result of inadequate training, insufficient time for PHC workers to address these problems, and inaccessible or non-existent referral pathways. ${ }^{19}$ Several studies indicate the ongoing need for culturally acceptable and evidence-based psychological treatment services for CMDs. ${ }^{10}$ High rates of maternal depression ${ }^{22,23}$ and depression in PLWHAs in South Africa ${ }^{24-26}$ require that HIV and maternal health services integrate mental health into their care. There is also a need for referral pathways for trauma, which is highly predictive of CMDs. ${ }^{27}$

\section{- Secondary care}

At this level, psychotropic medication is usually available in general hospital inpatient psychiatric units and outpatient facilities. ${ }^{11}$ However, a number of studies reveal that infrastructure and specialist personnel are mostly inadequate for providing 72 hour emergency management and observation in general hospitals, as required by the Mental Health Care Act. In particular there is insufficient training and support of PHC personnel in emergency management of MHCUs. ${ }^{10}$ Where they are able to, such as in the Western Cape, many MHCUs have been reported to by-pass the district hospital 72-hour observation period and are referred directly to tertiary facilities, in contravention of the Act. ${ }^{28}$ Referral information to secondary level care is also inadequate ${ }^{10}$ and there are poor information systems for monitoring the delivery of mental health care at secondary level. ${ }^{11}$

\section{- Tertiary care}

At this level, the research reports "revolving door" patterns of care, in which MHCUs who are discharged from tertiary facilities are frequently re-admitted due to inadequate care in the community. This has been attributed to poor treatment adherence and defaulting; early discharge due to bed shortages; and substance abuse. ${ }^{10}$ This is not assisted by ongoing attrition of tertiary hospital bed numbers. In general terms, there was a 7.7\% reduction in mental hospital beds across all provinces between 2000 and $2005^{11}$, and a drop in income in two thirds of psychiatric hospitals in KwaZulu-Natal in a 5-year period following 2002. ${ }^{29}$ This means that effectively there are less tertiary facilities available for a growing population of people in need of services. Yet resources saved from tertiary bed reductions have not been re-allocated to acute facilities at district or regional general hospitals, and mental hospitals remain the main form of inpatient care, with 56\% of beds located in these settings in 2005. ${ }^{11}$ There have also been reports of dehumanizing experiences and human rights abuses in these facilities. ${ }^{10}$

\section{Cross-cutting themes - HIV/AIDS}

There is a higher prevalence of mental disorders in PLWHAs $[7,24,26,30]$. Of particular concern are findings that depression and alcohol misuse compromise ART adherence and 
virological suppression. ${ }^{5,6,8}$ In addition, people with common mental disorders are more likely to engage in inappropriate behavior change strategies to prevent HIV infection ${ }^{31}$ and are more prone (particularly men) to high risk sexual behavior. ${ }^{32}$ In turn, people who are HIV positive are at increased risk for anxiety, depression, suicide and neuro-cognitive impairment. ${ }^{7}$ Research also reveals high rates of HIV among MHCUs with severe chronic disorders and a lack of training and support for mental health specialist staff in HIV prevention. ${ }^{10}$

\section{- Stigma and discrimination}

Stigma and discrimination against people living with mental illness is widespread ${ }^{33}$, and exacerbated by some traditional explanatory beliefs, being in receipt of a disability grant and having no employment. ${ }^{10}$ Stigma and discrimination have been shown to contribute to defaulting and social isolation. ${ }^{10}$ In other settings, involving MHCUs in planning the delivery of services, and increasing contact between community members and MHCUs have been shown to be effective in reducing stigma. ${ }^{34}$

\section{- Cultural congruence}

MHCUs with severe mental disorders often utilize both western and traditional healing systems, yet the research indicates that there is little cooperation between the two systems of healing. 10 Language and class differences have been identified as barriers to accessing psychological services, with translation services neither optimal nor desirable.10 A minority of people with CMDs (about one fifth) seek help from alternative healers including traditional healers and spiritual advisors. ${ }^{35}$

\section{- Resources}

The resources required to deliver mental health services (including human resources, service facilities and budgets) have been consistently shown to be inadequate. ${ }^{36}$ There is a substantial shortfall particularly in resources for child and adolescent mental health services. ${ }^{37}$ In three separate contracts for the national Department of Health, norms have been calculated for in-patient and community-based services for people with severe mental disorders ${ }^{36,38}$; and child and adolescent mental health services. ${ }^{37}$ Yet these norms have yet to be systematically implemented, and there is an urgent need to train district and provincial health planners in the use of the norms.

\section{- Information systems}

As a final cross-cutting theme, information systems that are required to routinely monitor and evaluate mental health services are largely inadequate. ${ }^{11}$ There is a particular need to develop indicators of routine mental health service delivery at primary and secondary care level, and to integrate these indicators into the district health management information system (DHIS).

\section{Recommendations}

\section{- Service development}

A number of recommendations have emerged from research conducted on mental health services in South Africa during the last 12 years. ${ }^{10}$ First, there is need for substantial new investment of resources to support 72-hour decentralized care in designated regional and district hospitals. Second, there is a need to strengthen identification and management of CMDs using task shifting within a stepped care approach at the primary health care facility level [39]. This requires training, support and supervision of general primary care staff, particularly in maternal and HIV clinics to identify CMDs, manage and refer where appropriate. The use of guidelines such as those provided by the World Health Organization's Mental Health Gap Action Programme (mhGAP) Intervention Guide $^{40}$, which has been adapted for South Africa and integrated into set of clinical guidelines (Primary Care 101) (1) $^{4}$ recommended. It is also vital that we harness trained lay counselors and community health workers (CHWs) to deliver manualized psychosocial intervention packages for specific conditions, under supervision from mental health specialists such as BPsych Counselors.

Third, at the community level, community-based PSR programmes need to be developed, harnessing task shifting and self-help strategies driven by NGOs, particularly MHCU organisations. Support for the existing network of NGOs affiliated to South African Federation for Mental Health is essential. Public education is needed to improve mental health literacy and reduce stigma and discrimination. Finally, we need to establish collaborative arrangements with traditional healers to promote culturally congruent care and referral systems between the two systems of healing.

\section{- Research}

The bulk of mental health research in South Africa during the last 12 years provides information on burden of mental illness and the status of mental health services. There is an urgent need to shift the research agenda to a focus on intervention studies, including economic evaluations. This needs to include evaluations of evidence-based culturally appropriate packages of care using a task shifting approach; and the human resource mix and costing for the delivery of integrated packages of care at district level. The latter includes training and supervisory needs, organizational arrangements that promote cultural congruence, and capacity development needs for staff.

\section{Conclusion}

The political momentum created by the national mental health summit of April 2012 gives reason for hope that mental health services may at last begin to be given the resources and policy priority they deserve. However, this is unlikely to make any real difference in the lives of people who live with mental illness, particularly those in poor and marginalized communities, unless there is a commitment to action. This requires concrete steps by all stakeholders, including the national and provincial Department of Health; other social sector Departments such as Education, Housing, Social Development and Justice; mental health professionals and general health workers at all levels; NGOs, including organisations led by MHCUs; and academic researchers and trainers. These steps need to be supported by a research programme focused on evaluating the costeffectiveness of culturally appropriate interventions. The time to act is now.

\section{References}

1. Mathers C, Loncar D. Projections of Global Mortality and Burden of Disease from 2002 to 2030. Public Library of Science Medicine 2006; 3.

2. Bradshaw D, Norman R, Schneider M. A clarion call for action based on refined DALY estimates for South Africa. South African Medical Journal 2007; 97: 438-440. 
3. Williams DR, Herman A, Stein DJ, Heeringa SG, Jackson PB, et al. Prevalence, Service Use and Demographic Correlates of 12-Month Psychiatric Disorders in South Africa: The South African Stress and Health Study. Psychological Medicine 2008; 38: 211-220.

4. Prince M, Patel V, Saxena S, Maj M, Maselko J, et al. No health without mental health. Lancet 2007; 370: 859-877.

5. Leserman J. Role of depression, stress, and trauma in HIV disease progression. Psychosom Med 2008; 70: 539-545

6. Gonzalez JS, Batchelder AW, Psaros C, Safren SA . Depression and HIVIAIDS treatment nonadherence: a review and meta-analysis. $J$ Acquir Immune Defic Syndr 201 1; 58: 181-187.

7. Joska JA, Fincham DS, Stein DJ, Paul RH, Seedat S. Clinical correlates of HIV-associated neurocognitive disorders in South Africa. AIDS Behav 2010; 14: 371-378.

8. Neuman MG, Schneider M, Nanau RM, Parry C. Alcohol Consumption, Progression of Disease and Other Comorbidities, and Responses to Antiretroviral Medication in People Living with HIV. AIDS Res Treat 2012; 2012: 751827.

9. Department of Health. White paper for the transformation of the health system in South Africa. Pretoria: Government Gazette; 1997.

10. Petersen I, Lund C. Mental health service delivery in South Africa from 2000 to 2010: One step forward, one step back. South African Medical Journal 2011; 101: 751-757.

11. Lund C, Kleintjes S, Kakuma R, Flisher A, the MRPC. Public sector mental health systems in South Africa: inter-provincial comparisons and policy implications. Social Psychiatry and Psychiatric Epidemiology 2010; 45: 393-404.

12. Flisher AJ, Lund C, Funk M, Banda M, Bhana A, et al. Mental health policy development and implementation in four African countries. Journal of Health Psychology 2007; 12: 505-516.

13. Lund C, Kleintjes S, Cooper S, Petersen I, Bhana A, et al. Challenges facing South Africa's mental health care system: stakeholders' perceptions of causes and potential solutions. International Journal of Culture and Mental Health 201 1; 4: 23-38.

14. Brekke JS, Long JD. Community-based psychosocial rehabilitation and prospective change in functional, clinical, and subjective experience variables in schizophrenia. Schizophrenia bulletin 2000; 26: 667-680

15. Kleintjes S, Lund C, Swartz L (In press) Organising for self-advocacy in mental health: Experiences from 7 African countries. African Journal of Psychiatry.

16. Botha U, Koen L, Joska J, Hering L, Oosthuizen P. Assessing the efficacy of a modified assertive community-based treatment programme in a developing country. BMC Psychiatry 2010; 10: 73.

17. Van Deventer C, Couper I. Evaluation of primary mental healthcare in North West province: A qualitative view. South African Journal of Psychiatry 2008; 14: 136-140

18. Breen A, Swartz L, Flisher AJ, et al. Experience of mental disorder in the context of basic service reforms: The impact of caregiving environments in South Africa. International Journal of Environmental Health Research 2007; 17: 327-334.

19. Petersen I, Bhana A, Campbell-Hall V, Mjadu S, Lund C, et al. Planning for district mental health services in South Africa: a situational analysis of a rural district site. Health Policy And Planning 2009; 24: 140-150.

20. Modiba P, Schneider H, Porteus KA, Gunnarson V. Profile of community mental health service needs in the Moretele District (North West Province) in South Africa. Journal of Mental Health Policy and Economics2001; 4: 189-196.

21. Sorsdahl K, Flisher AJ, Ward C, et al. The time is now: missed opportunities to address patient needs in community clinics in Cape Town, South Africa. Tropical Medicine \& International Health2010; 15 1218-1226
22. Rochat TJ, Tomlinson M, Barnighausen T, Newell ML, Stein A. The prevalence and clinical presentation of antenatal depression in rural South Africa. J Affect Disord 201 1; 135: 362-373.

23. Cooper PJ, Tomlinson M, Swartz L, Woolgar M, Murray L, et al. Postpartum depression and the mother-infant relationship in a South African peri-urban settlement. Br J Psychiatry 1999; 175: 554-558.

24. Freeman M, Nkomo N, Kafaar Z, Kelly K. Factors associated with prevalence of mental disorder in people living with HIVIAIDS in South Africa. AIDS Care 2007; 19: 1201-1209.

25. Spies G, Kader K, Kidd M, Smit J, Myer L, et al. Validity of the K-10 in detecting DSM-IV-defined depression and anxiety disorders among HIV-infected individuals. AIDS Care 2009; 21: 1163-1168.

26. Olley BO, Seedat S, Stein DJ. Persistence of psychiatric disorders in a cohort of HIVIAIDS patients in South Africa: a 6-month follow-up study. J Psychosom Res 2006; 61: 479-484.

27. Seedat S, Stein DJ, Jackson PB, Heeringa SG, Williams DR, et al. Life stress and mental disorders in the South African stress and health study. S Afr Med J 2009; 99: 375-382.

28. Lund C, Oosthuizen P, Flisher AJ, Emsley R, Stein DJ, et al. Pathways to Inpatient Mental Health Care Among People With Schizophrenia Spectrum Disorders in South Africa. Psychiatric Services 2010; 61: 235-240.

29. Burns JK, Jhazbhay K, Kidd M, Emsley RA . Causal attributions, pathway to care and clinical features of first-episode psychosis: a South African perspective. Int J Soc Psychiatry201 1; 57: 538-545.

30. Myer L, Smit J, Roux LL, Parker S, Stein DJ, et al. Common mental disorders among HIV-infected individuals in South Africa: prevalence, predictors, and validation of brief psychiatric rating scales. AIDS Patient Care STDS 2008; 22: 147-158.

31. Myer L, Stein DJ, Grimsrud AT, Herman A, Seedat S, et al. DSM-IVdefined common mental disorders: association with HIV testing, HIV-related fears, perceived risk and preventive behaviours among South African adults. S Afr Med J 2009; 99: 396-402.

32. Olley BO, Gxamza F, Seedat S, Theron H, Taljaard J, et al. Psychopathology and coping in recently diagnosed HIVIAIDS patients--the role of gender. S Afr Med J 2003; 93: 928-931

33. Kakuma R, Kleintjes S, Lund C, Drew N, Green A, et al. Mental health stigma: what is being done to raise awareness and reduce stigma in South Africa? African Journal of Psychiatry 2010; 13: 116-124.

34. Thornicroft G. Shunned: Discrimination against people with mental illness. London: Oxford University Press. 2006.

35. Sorsdahl K, Stein DJ, Grimsrud A, Seedat S, Flisher AJ, et al. Traditional healers in the treatment of common mental disorders in South Africa.J Nerv Ment Dis 2009; 197: 434-441.

36. Lund C, Flisher AJ. Norms for mental health services in South Africa. Social Psychiatry and Psychiatric Epidemiology 2006; 41: 587-594.

37. Lund C, Boyce G, Flisher AJ, Kafaar Z, Dawes A. Scaling up child and adolescent mental health services in South Africa: Human resource requirements and costs. Journal of Child Psychology and Psychiatry 2009; 50: 1121-1130

38. Lund C, Flisher AJ. A model for community mental health services in South Africa. Tropical Medicine \& International Health 2009; 14: 10401047.

39. Petersen I, Lund C, Bhana A, Flisher AJ, the Mental H, et al. A task shifting approach to primary mental health care for adults in South Africa: human resource requirements and costs for rural settings Health Policy And Planning 2012; 27(1): 42-51.

40. World Health Organization. mhGAP Intervention Guide. Geneva: World Health Organization. 2010

41. University of Cape Town Lung Institute . Primary Care 101. Pretoria: Department of Health, South Africa. 2012. 